\title{
A Revised Alverson and Carney Model for Estimating the Instantaneous Rate of Natural Mortality
}

\author{
CHANG-IK ZHANG \\ Pukyong National University, College of Fisheries Science, Department of Marine Production Management, \\ 599-1 Daeyeon 3-Dong, Nam-Gu, Busan 608-737, Korea
}

\author{
Bernard A. Megrey* \\ National Oceanic and Atmospheric Administration, National Marine Fisheries Service, \\ Alaska Fisheries Science Center, 7600 Sand Point Way NE, Seattle, Washington 98115, USA
}

\begin{abstract}
The Alverson and Carney (AC) model for estimating the instantaneous rate of natural mortality $(M)$ was reformulated to include $\beta$ and $t_{0}$ parameters. The revised Zhang and Megrey (ZM) model could be used for the estimation of $M$ instead of the AC model since von Bertalanffy and allometric growth parameters are readily available for most exploited fish stocks. It was determined from the new formulation that the ratios of the age at maximum biomass $\left(t_{\mathrm{mb}}\right)$ to the maximum age $\left(t_{\max }\right)$ for pelagic and demersal species were significantly different from 0.38 , the value originally proposed by Alverson and Carney. The ratios for these two ecological groups were 0.302 and 0.440 , respectively, and were significantly different. We examined the sensitivity and bias in $M$ from the new formulation relative to those from the AC model, which assumed that $\beta$ $=3.0$ and $t_{0}=0$. Estimates of $M$ from the AC model are most sensitive to the assumption that growth starts at $t_{0}=0$ when growth rates are high as well as to the $\beta$ and $t_{\mathrm{mb}}$ assumptions. The performance of the revised ZM model was evaluated by comparing calculated $M$ values from the two models based on a paired-sample $t$-test. The results of the two statistical analyses showed that the ZM model produced values of $M$ closer to published estimates than did the AC model. Thus, the ratio $t_{\mathrm{mb}} / t_{\max }$ for specific ecological groups should be used rather than Alverson and Carney's constant 0.38. Analyses of exploited stock dynamics might be conducted using the possible range of $M$ instead of the constant value. The range could be estimated from the ZM model using the mean ratio $t_{\mathrm{mb}} / t_{\max } \pm \mathrm{SD}$ to get the $t_{\mathrm{mb}}$ for each subgroup or by explicit variance calculations.
\end{abstract}

The instantaneous rate of natural mortality $(M)$ in exploited fish stocks is defined as the mortality created by all causes other than fishing. These include deaths from predation, cannibalism, disease, spawning stress, starvation, and senescence (Beverton and Holt 1957).

The value of $M$ is considered one of the two critical aspects of the dynamics of a single species, which, together with the relation between recruitment and adult stock, produces significant uncertainties in longterm stock assessment and the strategic advice given to managers using traditional approaches. The significance of the value of $M$ for management advice (e.g., on target values of fishing mortality, $F$ ) is well known to stock assessment scientists (Clark 1999) but has received less attention from the general fishery research community. One reason for this is that the degree of uncertainty in the values of $M$ currently being used is not always obvious. Many assessment techniques, such as virtual population assessments, require setting the value of $M$ early in the analysis, and the values used by assessment groups often become institutionalized mainly from customary use. Another reason is that it

\footnotetext{
* Corresponding author: bern.megrey@noaa.gov
}

Received October 10, 2005; accepted October 26, 2005 Published online May 30, 2006 is difficult to identify lines of research that offer good prospects for coming up with better estimates of $M$ (Gulland 1983). Therefore, at present, $M$ is considered an important but poorly quantified parameter in most mathematical models of fish stock dynamics (Vetter 1988; Quinn and Deriso 1999).

Direct measurements of $M$ are often difficult to obtain. Consequently, there have been many attempts to identify quantities that can be directly or indirectly related to $M$ and that are easier to estimate (Sparre et al. 1989). Several attempts along these lines have been made to estimate $M$ indirectly using reproduction indices (Rikhter and Efanov 1976; Gunderson and Dygert 1988; Gunderson 1997) or life history parameters (Alverson and Carney 1975; Pauly 1980).

Both the instantaneous rate of natural mortality and the curvature of growth on age are fundamental population-dynamic measures of fish. Growth and mortality may be correlated in an empirically useful way, namely, fast-growing fish tend to have higher mortality rates. The von Bertalanffy curvature parameter, $K$, which describes the rate at which the maximum size is approached, is linked to the longevity of the fish (Beverton and Holt 1959; Beverton 1992), and longevity is related to mortality (Tanaka 1960; Holt 1965; Saville 1977; Hoenig 1983; Alagaraja 1984). Ralston (1987) argued that $M$ can simply be predicted 
with $K$, the former being roughly twice the latter, based on a review of the snapper (Lutjanidae) and grouper (Serranidae) literature. More recently, Jensen (1996) concluded that $K$ alone was adequate for prediction. He proposed the formula $M=1.60 K$ based on the data for $K$ and $M$ on 175 different species provided by Pauly (1980). As a rough generalization, a long-lived fish approaches its limiting size relatively slowly, whereas a short-lived fish grows rapidly. Thus, fish species with high $K$ values usually have high $M$ values, and vice versa. A slowly growing species cannot bear high natural mortality, because if it did, it would never reach maturity and would soon go extinct.

Alverson and Carney (1975) assumed populations that had an exponential decay function describing their abundance through time and a von Bertalanffy growth schedule. They derived an empirical equation relating $M$ to the maximum age and the growth parameter $K$. They solved the relationship between maximum age and critical age (the age at which the biomass of the cohort is maximum) using 63 reported stocks. However, they assumed that fish length was zero at age zero (i.e., eliminating the parameter $t_{0}$ ). Alverson and Carney (1975) stated that their empirical relationship would require future adjustments to accommodate negative values of $t_{0}$. Alverson and Carney (1975) also assumed isometric growth, that is, that weight is proportional to the cube of length. Quinn and Deriso (1999) indicate that the cubic relationship does not usually fit actual data well, and they also explicitly caution that the Alverson and Carney approach has not been strictly reevaluated in terms of the allometric growth model.

In spite of the simplifying assumptions, the Alverson and Carney model is still widely used to estimate $M$, and most population dynamics textbooks introduce this model as one of the standard methods (Sparre et al. 1989; Zhang 1991; Quinn and Deriso 1999). Moreover, a number of applied studies continue to rely on this model to estimate $M$ (Efimov 1984; Megrey et al. 1990; Wakabayashi and Watanabe 1990; Quinn and Szarzi 1993; Zhang et al. 1998a; Wilderbuer and Zhang 1999).

In this paper, we have three objectives: (1) to reformulate the Alverson and Carney empirical relationship for estimating $M$ to include the $\beta$ and $t_{0}$ parameters; (2) to examine the sensitivity and bias of $M$ to the assumptions that $\beta=3.0$ and $t_{0}=0$; and (3) to reexamine Alverson and Carney's use of a constant 0.38 in relating the maximum age to the critical age and test whether the 0.38 multiplier is generally applicable for ecologically different groups of fishes, since the more $r$-strategic pelagic species could have different life history characteristics from the more $K$-strategic demersal species.

\section{Methods}

Derivation of the reformulation.-The exponential decay curve of Baranov (1918), which describes the decrease in abundance through time due to mortality, is given by

$$
N_{t}=N_{0} e^{-M t}
$$

where $N_{t}$ is the abundance at time $t, N_{0}$ is the abundance at $t=0$, and $M$ is the rate of natural mortality.

The growth of an animal, in length, can be represented by the von Bertalanffy model (von Bertalanffy 1938),

$$
L_{t}=L_{\infty}\left[1-e^{-K\left(t-t_{0}\right)}\right]
$$

where $L_{t}$ is the mean length of the fish at age $t, L_{\infty}$ is the mean asymptotic length (i.e., the length it would ultimately reach at an infinitely old age), $K$ is the Brody growth rate per unit of time, and $t_{0}$ is the theoretical age at which the fish would have length zero if it had always grown as described by equation (2). Theoretically, $t_{0}$ is rarely equal to zero, and it is usually less than zero (Quinn and Deriso 1999).

Fish growth can be expressed in terms of length or weight since the three dimensions making up a fish's body_length, width, and depth—all change over time. Under isometric assumptions, weight at age can be written as

$$
W_{t}=\alpha L_{t}^{3}
$$

where $L_{t}$ represents length and $W_{t}$ represents weight, both at age $t$. With fish, the isometric relationship typically does not hold (Quinn and Deriso 1999), so equation (3) needs to be generalized to the allometric model, written as

$$
W_{t}=\alpha L_{t}^{\beta}
$$

which assumes that growth in weight is proportional to some power of length. Equations (2) and (4) can be combined to express growth in weight through time as follows:

$$
W_{t}=W_{\infty}\left[1-e^{-K\left(t-t_{0}\right)}\right]^{\beta},
$$

where $W_{t}$ is the mean weight of the fish at age $t$ and $W_{\infty}$ is the mean asymptotic weight.

Biomass at age $t, B_{t}$, is obtained by multiplying equation (1) by equation (5),

$$
B_{t}=N_{t} W_{t}=N_{0} W_{\infty} e^{-M t}\left[1-e^{-K\left(t-t_{0}\right)}\right]^{\beta} .
$$

The instantaneous change in biomass with respect to age $t$ is the time derivative of equation (6), namely,

$$
\begin{aligned}
\frac{d B_{t}}{d t}= & \beta K e^{K t_{0}} N_{0} W_{\infty} e^{-(M+K) t}\left[1-e^{-K\left(t-t_{0}\right)}\right]^{\beta-1} \\
& -M N_{0} W_{\infty} e^{-M t}\left[1-e^{-K\left(t-t_{0}\right)}\right]^{\beta} .
\end{aligned}
$$

By setting equation (7) to zero and $t=t_{\mathrm{mb}}$, the critical 
age (the time that the cohort achieves its maximum biomass) is

$$
\hat{M}_{\mathrm{ZM}}=\frac{\beta K}{e^{K\left(t_{\mathrm{mb}}-t_{0}\right)}-1},
$$

where $t_{\mathrm{mb}}=C_{i} \cdot t_{\max }$. Here, $t_{\max }$ is the maximum age observed in the population (Beverton and Holt 1959; Alagaraja 1984). Since the ratio of the critical age to the maximum age could be different from a constant 0.38 for different ecological groups (especially for pelagic versus demersal species), the constant for each specific ecological group $i, C_{i}$ is defined. In this study, $i$ represents either the pelagic group or the demersal group. Equation (8) (hereafter referred to as the Zhang and Megrey model, or ZM model) indicates that natural mortality $M$ can be expressed as a function of the growth rate $(K)$, the power parameter of the length -weight relationship $(\beta)$, the time the growth curve begins $\left(t_{0}\right)$, and the critical age $\left(t_{\mathrm{mb}}\right)$.

Equation (8) can be rearranged to provide an estimate of the time at which a cohort maximizes its weight and the animal has reached critical age, namely,

$$
t_{\mathrm{mb}}=\frac{\log _{e}\left(\frac{\beta \mathrm{\beta}^{K_{t_{0}}}}{\hat{\mathrm{M}}_{\mathrm{ZM}}}+e^{K t_{0}}\right)}{K} .
$$

Equation (8) is an extension to the formulation of Alverson and Carney (1975; hereafter referred to as the Alverson and Carney model, or AC model),

$$
\hat{M}_{\mathrm{AC}}=\frac{3 K}{e^{K t_{\mathrm{mb}}}-1},
$$

where Alverson and Carney assumed that the proportionality relating $t_{\mathrm{mb}}$ to $t_{\max }$ is constant for all species defined by $t_{\mathrm{mb}}=0.38 \cdot t_{\max }$.

The variance of $M$ can be approximated from equation (8) using the delta method (Seber 1982), as follows:

$$
\begin{aligned}
& \operatorname{var}(\hat{M})=\left(\frac{\partial \hat{M}}{\partial \beta}\right)^{2} \cdot \operatorname{var}(\beta)+\left(\frac{\partial \hat{M}}{\partial K}\right)^{2} \cdot \operatorname{var}(K) \\
& +\left(\frac{\partial \hat{M}}{\partial t_{0}}\right)^{2} \cdot \operatorname{var}\left(t_{0}\right)+\left(\frac{\partial \hat{M}}{\partial t_{\mathrm{mb}}}\right)^{2} \cdot \operatorname{var}\left(t_{\mathrm{mb}}\right) \\
& +2 \operatorname{cov}(\beta, K) \frac{\partial \hat{M}}{\partial \beta} \frac{\partial \hat{M}}{\partial K}+2 \operatorname{cov}\left(\beta, t_{0}\right) \frac{\partial \hat{M}}{\partial \beta} \frac{\partial \hat{M}}{\partial t_{0}} \\
& +2 \operatorname{cov}\left(\beta, t_{\mathrm{mb}}\right) \frac{\partial \hat{M}}{\partial \beta} \frac{\partial \hat{M}}{\partial t_{\mathrm{mb}}}+2 \operatorname{cov}\left(K, t_{0}\right) \frac{\partial \hat{M}}{\partial K} \frac{\partial \hat{M}}{\partial t_{0}} \\
& +2 \operatorname{cov}\left(K, t_{\mathrm{mb}}\right) \frac{\partial \hat{M}}{\partial K} \frac{\partial \hat{M}}{\partial t_{\mathrm{mb}}}+2 \operatorname{cov}\left(t_{0}, t_{\mathrm{mb}}\right) \frac{\partial \hat{M}}{\partial t_{0}} \frac{\partial \hat{M}}{\partial t_{\mathrm{mb}}}
\end{aligned}
$$

This requires evaluating the partial derivatives of equation (8) with respect to $t_{0}, t_{\mathrm{mb}}, K$, and $\beta$, namely,

$$
\begin{array}{cc}
\frac{\partial \hat{M}}{\partial t_{0}}=\frac{\beta K^{2} e^{K\left(t_{\mathrm{mb}}-t_{0}\right)}}{\left[e^{K\left(t_{\mathrm{mb}}-t_{0}\right)}-1\right]^{2}} \quad(11 a) \\
\frac{\partial \hat{M}}{\partial t_{\mathrm{mb}}}=\frac{-\beta K^{2} e^{K\left(t_{\mathrm{mb}}-t_{0}\right)}}{\left[e^{K\left(t_{\mathrm{mb}}-t_{0}\right)}-1\right]^{2}} \quad(11 b) \\
\frac{\partial \hat{M}}{\partial K}=\frac{\beta\left[e^{K\left(t_{\mathrm{mb}}-t_{0}\right)}-K t_{\mathrm{mb}} e^{K\left(t_{\mathrm{mb}}-t_{0}\right)}+K t_{0} e^{K\left(t_{\mathrm{mb}}-t_{0}\right)}-1\right]}{\left[e^{K\left(t_{\mathrm{mb}}-t_{0}\right)}-1\right]^{2}} \\
\frac{\partial \hat{M}}{\partial \hat{\beta}}=\frac{K}{e^{K\left(t_{\mathrm{mb}}-t_{0}\right)}-1} . & (11 c)
\end{array}
$$

Sensitivity of estimates of $M$ to $\beta$ and $t_{0}$ assumptions.Estimates of $M$ were calculated according to the AC model $\left(\hat{M}_{\mathrm{AC}}\right.$ from equation 10$)$ and the $\mathrm{ZM}$ model $\left(\hat{M}_{\mathrm{ZM}}\right.$ from equation 8$)$ using values of $K=0.1$ and $t_{\max }$ $=20$. The values of $t_{\mathrm{mb}}$ for both models were calculated from the formula $t_{\mathrm{mb}}=C_{i} \cdot t_{\max }$. For demersal species, values of $C_{i}=0.38\left(t_{\mathrm{mb}}=7.6\right)$ and $C_{i}=0.44\left(t_{\mathrm{mb}}=8.8\right)$ are used in the $\mathrm{AC}$ and $\mathrm{ZM}$ models, respectively. For pelagic species, values of $C_{i}=0.38$ and $C_{i}=0.302\left(t_{\mathrm{mb}}\right.$ $=6.04)$ are used.

Sensitivity of estimates of $M$ to $\beta$ and $t_{m b}$ assumptions. - Estimates of $M$ were calculated according to the $\mathrm{AC}$ and $\mathrm{ZM}$ models using values of $t_{\mathrm{mb}}$ ranging from 5.0 to $20.0, \beta$ from 2.5 to 3.5 , and $t_{0}$ from -3.0 to +3.0 . The percent difference in the estimates of $M$ from the $\mathrm{AC}$ and $\mathrm{ZM}$ models was computed according to the formula

$$
\text { Percent difference }=\frac{\hat{M}_{\mathrm{ZM}}-\hat{M}_{\mathrm{AC}}}{\hat{M}_{\mathrm{AC}}} \times 100 .
$$

Two cases were considered. In the first, $M$ values from the $\mathrm{ZM}$ and $\mathrm{AC}$ models were calculated as a function of $\beta$ and $t_{\mathrm{mb}}$, with $t_{0}$ fixed at 3.0 for a fastgrowing $(K=1.5)$ and a slow-growing $(K=0.1)$ fish. In the second, $M$ values from the $\mathrm{ZM}$ and AC models were calculated as a function of $\beta$ and $t_{0}$, with $t_{\mathrm{mb}}$ fixed at 5.0 for a fast-growing and a slow-growing fish.

Data.-The data used to evaluate the relationships described earlier were based on a compilation of data extracted from the FishBase 98 database (Frose and Pauly 1998), those summarized by Ault et al. (1998) and Ault et al. (2005), and those from other data sources known to the authors (Table 1). In the following sections, estimated values of $\hat{M}_{\mathrm{AC}}$ and $\hat{M}_{\mathrm{ZM}}$ are compared with those reported in FishBase, which we refer to as the "true" values of $M\left(M_{\text {true }}\right)$. Records from FishBase that contained all five values were extracted and screened by eliminating duplicate records. A total of 91 values of $K, t_{0}, M_{\text {true }}, \beta$, and maximum age $\left(t_{\max }\right)$ 
were assembled. There were not many fish stocks that had all of the parameters required for the calculations necessary in this study. Multiple length-weight conversion parameters and values of $t_{\max }$ were available from FishBase for each species, so the values of $\beta$ and $t_{\max }$ reported in Table 1 represent the arithmetic average and the maximum value, respectively.

We consider data from FishBase suitable for our analysis since all of the life history parameters contained in FishBase are independent, that is, they were not estimated using rules of thumb or empirical models linking $M$ to a predictor variable or variables such as those presented by Pauly (1980). Indeed, Frose and Pauly (1998) explicitly point out that the data in FishBase are appropriate for deriving new empirical models.

We use data for the Australian gemfish (Rowling 1999) to illustrate the utility of our new formulation. Detailed growth data on this species are shown in Table 1. Data on the variances of $K, t_{0}$, and $\beta$ are from Rowling (1999), and those for $t_{\max }$ are from Rowling (1997). Even though we had only one observation of $t_{\max }$ for this example, we calculated a variance for this variable by assuming that its coefficient of variation was $10 \%$ and that its mean value was 16 . We believe that this assumption is reasonable, as the maximum age of a fish should be known with great precision. Using the delta method, the variance of $t_{\mathrm{mb}}$ was calculated as

$$
\operatorname{var}\left(t_{\mathrm{mb}}\right)=C_{i}^{2} \operatorname{var}\left(t_{\max }\right) .
$$

Evaluation of the relationship of $t_{m b}$ to the maximum age for different ecological groups.-For the estimation of $t_{\mathrm{mb}}$, the data in Table 1 were disaggregated into two ecological subgroups defined as pelagic species (31 stocks) and demersal species (60 stocks). Using the values of $K, M_{\text {true }}, \beta$, and $t_{0}$ from Table $1, t_{\mathrm{mb}}$ was estimated from equation (9) and then the ratio $t_{\mathrm{mb}} / t_{\max }$ was calculated. Statistical tests on the mean ratios for the pelagic and demersal groups were performed to assess whether they were statistically different for the two groups as well as different from 0.38 , the value proposed by Alverson and Carney. In technical terms, a two-tailed $t$-test was used to test the null hypotheses that $\mu_{\text {pelagic }}=\mu_{\text {demersal }}=0.38$.

To evaluate the performance of the ZM model, $M$ values calculated from equations (8) and (10) were compared with $M_{\text {true }}$ by means of a paired-sample $t$ test. A paired two-tailed $t$-test was used to test the null hypothesis that the difference between $\hat{M}_{\mathrm{AC}}$ or $\hat{M}_{\mathrm{ZM}}$ and $M_{\text {true }}$ was zero.

\section{Results}

Sensitivity of Estimates of $M$ to $\beta$ and $t_{0}$ Assumptions

A comparison of the results from estimating $M$ from the $\mathrm{AC}$ and $\mathrm{ZM}$ models is shown in Figure 1 for demersal and pelagic species. In the AC model, $\beta=$
$3.0, t_{0}=0$, and $\hat{M}_{\mathrm{AC}}=0.2636$ over the entire range of $\beta$ and $t_{0}$ and is shown as a plane. The variable $\hat{M}_{\mathrm{ZM}}$ is a nonlinear function of $\beta$ and $t_{0}$ and is shown as a curvilinear response surface. Where the two surfaces intersect corresponds to values of $\beta$ and $t_{0}$ where $\hat{M}_{\mathrm{AC}}$ $=\hat{M}_{\mathrm{ZM}}$.

The AC model did not show any difference between pelagic and demersal species because the $C_{i}$ value did not differ between these ecological groups. However, the ZM model showed that demersal species, which generally grow slowly, have lower mortalities than faster-growing pelagic species. The response surfaces from both models have the same shape for both ecological groups. The ZM model scales differently in the response of $M$ because different values of $C_{i}$ lead to different values of $t_{\mathrm{mb}}$. For demersal species, the AC model generally produces higher values of $M$ than the ZM model over a broad range of $\beta$ and $t_{0}$, but for pelagic species, the AC model generally produces lower values of $M$.

Sensitivity of Estimates of $M$ to $\beta$ and $t_{\mathrm{mb}}$ Assumptions

A comparison of the percent difference (calculated from equation 12) in estimates of $M$ from the two models is shown in Figure 2. The percent difference is shown as a function of $\beta$ and $t_{\mathrm{mb}}$ given $t_{0}=3$ for a fastgrowing $(K=1.5$; panel $\mathrm{A})$ and slow-growing fish $(K=$ 0.1 ; panel B) and as a function of $\beta$ and $t_{0}$ given $t_{\mathrm{mb}}=$ 5.0 for a fast-growing (panel C) and slow-growing fish (panel D). Specific values of $\hat{M}_{\mathrm{ZM}}, \hat{M}_{\mathrm{AC}}$, and the percent difference given below are written as a function of the parameters (i.e., $\hat{M}_{\mathrm{ZM}}\left[t_{\mathrm{mb}}, \beta\right], \hat{M}_{\mathrm{AC}}\left[t_{\mathrm{mb}}, \beta\right]$, and $\left.\% \operatorname{diff}\left[t_{\mathrm{mb}}, \beta\right]\right)$.

In most cases the $\mathrm{ZM}$ model produced higher values of $M$ than the AC model. The percent differences were higher in the case of fast-growing fish (panels A, C). For example, when $\beta$ and $t_{\mathrm{mb}}$ varied and $K$ and $t_{0}$ were fixed at 1.5 and 3, respectively, $\hat{M}_{\mathrm{ZM}}(5,3.5)=0.2719$, $\hat{M}_{\mathrm{AC}}(5,3.5)=0.00249$, and $\% \operatorname{diff}(5,3.5)=11,000$. When $\hat{M}_{\mathrm{ZM}}(10,2.5)=0.0001219, \hat{M}_{\mathrm{AC}}(10,2.5)=1.5$ $\times 10^{-6}$, and $\% \operatorname{diff}(10,2.5)=7,522$. The $\mathrm{ZM}$ model generally produced higher values of $M$ than the AC model. In the fast-growing case (panel A), $\hat{M}_{\mathrm{ZM}}$ was more responsive to changes in $\beta$ and mildly responsive to changes in $t_{\mathrm{mb}}$, resulting in large positive percent differences. In the slow-growing case (panel B), $\hat{M}_{\mathrm{ZM}}$ was responsive to both $\beta$ and $t_{\mathrm{mb}}$, still being more responsive to changes in $\beta$ and generating lower values of percent difference.

In the situation where $\beta$ and $t_{0}$ varied and $K$ and $t_{\mathrm{mb}}$ were fixed (panels $\mathrm{C}, \mathrm{D}$ ), the AC model produced a simple plane with $\hat{M}_{\mathrm{AC}}=0.0249$. Percent difference was very responsive to changes to $t_{0}$, especially for positive values of $t_{0}$. The response to $\beta$ was minor at 
TABLE 1.-Life history parameters used in this study (see text for definitions). Values of $t_{\mathrm{mb}}$ were calculated from equation 9 using $M_{\text {true }}$ as the value of natural mortality. References are keyed to the numbered list that follows the table.

\begin{tabular}{|c|c|c|c|c|c|c|c|c|c|c|c|c|c|}
\hline \multirow[b]{2}{*}{ Species } & \multicolumn{5}{|c|}{ Published values } & \multicolumn{3}{|c|}{ Calculated values } & \multicolumn{5}{|c|}{ Reference } \\
\hline & $t_{\max }$ & $K$ & $t_{0}$ & $M_{\text {true }}$ & $\beta$ & $t_{\mathrm{mb}}$ & $M_{\mathrm{ZM}}$ & $M_{\mathrm{AC}}$ & $t_{\max }$ & $K$ & $T_{0}$ & $M_{\text {true }}$ & $\beta$ \\
\hline \multicolumn{14}{|c|}{ Pelagic } \\
\hline \multirow{2}{*}{ Atlantic herring Clupea harengi } & 25 & 0.33 & -1.66 & 0.20 & 3.033 & 3.77 & 0.051 & 0.045 & 2 & 2 & 2 & 1 & \\
\hline & 25 & 0.44 & -1.27 & 0.16 & 3.033 & 3.81 & 0.028 & 0.021 & 2 & 2 & 2 & 3 & 2 \\
\hline Pacific herring Clupea pallasii & 15 & 0.16 & -2.49 & 0.42 & 3.27 & 2.57 & 0.253 & 0.322 & 4 & 4 & 4 & 4 & 4 \\
\hline \multirow{2}{*}{ Blackfin tuna Thunnus atlanticus } & 5 & 0.33 & -1.57 & 0.67 & 3.041 & 1.20 & 0.570 & 1.135 & 5 & 5 & 5 & 6 & 5 \\
\hline & 5 & 0.330 & -1.57 & 0.67 & 3.100 & 1.24 & 0.581 & 1.135 & 26 & 5 & 5 & 6 & 25 \\
\hline \multirow[t]{2}{*}{ King mackerel Scomberomorus cavalla } & 14 & 0.14 & -2.08 & 0.37 & 2.994 & 3.33 & 0.296 & 0.380 & 7 & 7 & 7 & 7 & 7 \\
\hline & 14 & 0.28 & -1.17 & 0.37 & 2.994 & 3.06 & 0.238 & 0.245 & 7 & 7 & 7 & 7 & 7 \\
\hline Yellowfin tuna Thunnus albacares & 5 & 0.45 & -0.75 & 0.80 & 2.881 & 1.39 & 0.736 & 0.999 & 8 & 8 & 8 & 8 & 8 \\
\hline & 5 & 0.327 & -1.02 & 0.80 & 2.981 & 1.42 & 0.758 & 1.139 & 8 & 29 & 28 & 27 & 27 \\
\hline Cutthroat trout Oncorhynchus clarkii & 9 & 0.16 & -0.38 & 0.30 & 3.032 & 5.63 & 0.757 & 0.659 & 9 & 9 & 9 & 9 & \\
\hline Pacific sardine Sardinops sagax caeruleus & 13 & 0.4 & -0.59 & 0.15 & 3.00 & 4.90 & 0.237 & 0.193 & 10 & 10 & 10 & 10 & 10 \\
\hline & 13 & 0.4 & -2.1 & 0.45 & 3.00 & 1.15 & 0.119 & 0.193 & 10 & 10 & 10 & 10 & 10 \\
\hline Australian salmon Arripis trutta & 26 & 0.38 & -0.07 & 0.80 & 2.80 & 2.16 & 0.055 & 0.027 & 11 & 11 & 11 & 11 & 11 \\
\hline & 26 & 0.3 & -0.17 & 0.80 & 2.80 & 2.22 & 0.084 & 0.049 & 11 & 11 & 11 & 11 & 11 \\
\hline Atlantic thread herring Opisthonema oglinum & 8 & 0.249 & -1.07 & 0.55 & 3.14 & 2.48 & 0.567 & 0.660 & 12 & 13 & 13 & 12 & 13 \\
\hline South American pilchard ${ }^{\text {a }}$ Sardinops sagax sagax & 5.5 & 0.358 & -0.24 & 0.79 & 2.83 & 2.07 & 1.041 & 0.965 & 14 & 14 & 14 & 14 & 15 \\
\hline European anchovy Engraulis encra & 5 & 0.380 & -0.93 & 0.81 & 3.41 & 1.58 & 0.850 & 1.077 & 16 & 16 & 16 & 16 & 16 \\
\hline chard Sardina pilchardus & 8 & 0.820 & -0.129 & 0.50 & 3.27 & 2.13 & 0.381 & 0.222 & 20 & 18 & 18 & 7 & 19 \\
\hline Albacore Thunnus alalunga & 6 & 0.262 & -1.31 & 0.20 & 2.877 & 4.65 & 0.596 & 0.962 & 24 & 22 & 22 & 21 & 23 \\
\hline a Katsuwonus pe & 4 & 0.110 & -1.97 & & 3.150 & 1.91 & 0.829 & & 28 & 5 & 5 & 6 & 25 \\
\hline hunnus thynnus & 13 & 0.162 & -1.12 & 0 . & 3.0 & 9.81 & 0.386 & 03 & 8 & 29 & 29 & 30 & 31 \\
\hline unnus obesus & 11 & 0.106 & -1.1 & 0. & 2. & 4.36 & 11 & & 33 & 32 & 32 & 33 & 34 \\
\hline dine Sardinops melanostictus & 9 & 0.467 & -0.09 & 0.49 & 3.615 & 3.10 & 0.624 & 0.356 & 35 & 36 & 36 & 35 & 37 \\
\hline ololabis saira & 2 & 0.340 & -1.19 & 1.60 & 3.220 & 0.34 & & & 40 & 38 & 38 & 38 & 39 \\
\hline mo salar & 6 & 0.288 & -0.46 & 1.1 & & 1.55 & 0.937 & & 8 & 43 & 43 & 41 & 42 \\
\hline Paci & 12 & 0.490 & 0 & 0 . & & 3.39 & & & 47 & 44 & 44 & 15 & 46 \\
\hline Greenback horse mackerel Trachurus declivis & 16 & 0.200 & -0.87 & 0.6 & 3.050 & 2.37 & 0.287 & 0.253 & 49 & 48 & 48 & 48 & 49 \\
\hline Jack & 30 & 0.093 & -2 & & 3.2 & 9.80 & & & 51 & 50 & 50 & 1 & 52 \\
\hline Narro & 14 & 0.250 & -1. & 0. & & 2.17 & 0.211 & 0 & 52 & 52 & 52 & 52 & 52 \\
\hline Nort & 7 & 0.349 & -0 & & & 2.11 & & & 56 & 53 & 53 & 4 & 55 \\
\hline chovy Engraulis $j$ & 3 & 1.586 & 0.001 & 0.80 & 3.6 & 1.34 & 1.829 & 0.933 & 60 & 57 & 57 & 8 & 59 \\
\hline & & & Demersal & & & & & & & & & & \\
\hline$A$ & 23 & 0.19 & -0.042 & 0.17 & 3 & 7.76 & 98 & 4 & 61 & 61 & 51 & 61 & \\
\hline Eurol & 10 & 0.19 & -0.85 & 0.20 & 8 & 6.31 & 0.338 & 38 & 62 & 62 & 62 & 62 & 62 \\
\hline Crimson jobfish Pristipomoides filamen & 18 & 0.15 & -1.67 & 0.25 & 2.653 & 4.68 & 0.124 & 0.251 & 63 & 63 & 63 & 63 & 63 \\
\hline Red snapper Lutjanus campechanus & 10 & 0.2 & & & & 6.85 & & & 64 & 64 & 64 & 4 & 64 \\
\hline & 16 & 0.162 & -0.01 & 0.19 & 20 & 7.75 & 0.224 & 0.290 & 65 & 65 & 65 & 55 & 65 \\
\hline B & 10 & 0.097 & -1.728 & 0.30 & 2.815 & 4.94 & 0.336 & 0.653 & 65 & 65 & 65 & 65 & 65 \\
\hline Inti & 9 & 0.084 & -2.896 & 0.23 & 2.974 & 5.86 & 0.321 & 0.757 & 65 & 65 & 65 & 65 & 65 \\
\hline & 20 & 0.16 & -0.30 & & & 8.76 & & & 65 & 65 & 65 & 5 & 65 \\
\hline Dog & 9 & 0.1 & -2.00 & 0.333 & 2 & 4.19 & 0.351 & 0.736 & 65 & 65 & 65 & 5 & 5 \\
\hline Gra & 10 & 0.136 & -0.863 & 0.30 & 2.881 & 5.28 & 0.375 & 0.603 & 65 & 65 & 65 & 65 & 65 \\
\hline-5 onatis & 10 & 0.097 & -1.728 & 0.30 & 2.815 & 4.94 & 0.336 & 0.653 & 65 & 65 & 65 & 65 & 5 \\
\hline $\mathrm{Ma}$ & 10 & 0.097 & -1.728 & 0.3 & & 4.78 & 0.325 & & 5 & 65 & 65 & 5 & 5 \\
\hline & 14 & 0.129 & -0.738 & 0.214 & 30 & 7.29 & 0.271 & 2 & 65 & 65 & 65 & 5 & 5 \\
\hline Schoo & 12 & 0.18 & 0.00 & 0.25 & 2.978 & 6.36 & 0.338 & 0.424 & 65 & 65 & 65 & 65 & 65 \\
\hline er Lutianus vivanus. & 9 & 0.092 & -2.309 & 0.23 & & 6.46 & 0.366 & 0.746 & 65 & 65 & 65 & 55 & 65 \\
\hline Ven & 1 & 0.206 & 0.111 & & & 6.38 & & & 5 & 65 & 65 & 5 & 5 \\
\hline Stri & 11 & 0.15 & -2.64 & 0.2 & 6 & 4.18 & 0.215 & 6 & 65 & 65 & 65 & 5 & 5 \\
\hline Epinephelus drum & 25 & 0.13 & -1.01 & 0.20 & 3.073 & 7.43 & 0.106 & 0.160 & 66 & 66 & 66 & 66 & 66 \\
\hline Snowy grouper Epinephelus niveatus & 27 & 0.09 & -1.01 & 0.18 & 2.843 & 8.82 & 0.117 & & 67 & 67 & 67 & 67 & 7 \\
\hline & 15 & 0.113 & -0.915 & 0. & & 10.29 & & & 65 & 65 & 65 & 5 & 5 \\
\hline & 20 & 0.16 & -0.30 & $0.1-1$ & 3.205 & 8.99 & 0.156 & 2 & 65 & 65 & 65 & 55 & 5 \\
\hline s fulva & 17 & 0.145 & -1.08 & 0.18 & 2.57 & 6.66 & 0.152 & 0.280 & 65 & 65 & 65 & 65 & 65 \\
\hline a microlepis & 13 & 0.149 & -0.802 & 0.20 & 3.031 & 7.13 & 0.275 & 0.411 & 65 & 65 & 65 & 55 & 65 \\
\hline 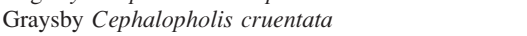 & 15 & 0.13 & -0.94 & 0.20 & 3.044 & 7.46 & 0.238 & 0.355 & 65 & 65 & 65 & 5 & 5 \\
\hline & 37 & & -3 & & & 16.65 & & & 65 & 65 & 65 & 55 & 5 \\
\hline er Epinephelus striatus & 17 & 0.145 & -1.08 & 0.18 & 3.229 & 7.76 & 0.190 & 0.280 & 65 & 65 & 65 & 65 & 65 \\
\hline er Epinephelus morio & 17 & 0.153 & -0.099 & 0.18 & 3.035 & 8.24 & 0.212 & 0.272 & 65 & 65 & 65 & 65 & 65 \\
\hline & 17 & 0.207 & -0.831 & 0. & & 5.87 & & 0.221 & 65 & 65 & 65 & 5 & \\
\hline Ro & 12 & 0.191 & -2.16 & 0.25 & & 4.10 & & & 65 & 65 & 65 & 65 & 65 \\
\hline Scamp Mycteroperca phenax & 21 & 0.126 & -1.357 & 0.143 & 2.993 & 8.89 & 0.135 & 0.218 & 65 & 65 & 65 & 65 & 65 \\
\hline Speckled hind & 15 & 0.13 & -1.01 & 0.20 & 3.073 & 7.43 & 0.237 & 0.355 & 65 & 65 & 65 & 65 & 65 \\
\hline & 41 & 0.054 & -3.616 & 0.08 & 2.98 & 16.80 & 0.073 & 0.123 & 65 & 65 & 65 & 65 & 65 \\
\hline & 15 & 0.17 & 0.0 & 0.1 & 2.98 & 7.88 & 0.245 & 0.312 & 65 & 65 & 65 & 65 & 65 \\
\hline Yellowfin grouper Mycteroperca venenosa & 15 & 0.17 & 0.00 & 0.18 & 2.98 & 7.88 & 0.245 & 0.312 & 65 & 65 & 65 & 65 & 65 \\
\hline
\end{tabular}


TABLE 1.-Continued.

\begin{tabular}{|c|c|c|c|c|c|c|c|c|c|c|c|c|c|}
\hline \multirow[b]{2}{*}{ Species } & \multicolumn{5}{|c|}{ Published values } & \multicolumn{3}{|c|}{ Calculated values } & \multicolumn{5}{|c|}{ Reference } \\
\hline & $t_{\max }$ & $K$ & $t_{0}$ & $M_{\text {true }}$ & $\beta$ & $t_{\mathrm{mb}}$ & $M_{\mathrm{ZM}}$ & $M_{\mathrm{AC}}$ & $t_{\max }$ & $K$ & $T_{0}$ & $M_{\text {true }}$ & \\
\hline Pollock Pollachius virens & 10 & 0.13 & -0.90 & 0.30 & 3.01 & 5.52 & 0.395 & 0.610 & 68 & 68 & 68 & 68 & 68 \\
\hline Rough sculpin Cottus asperrimus & 5 & 0.18 & -1.42 & 0.56 & 3.01 & 2.34 & 0.590 & 1.324 & 69 & 69 & 69 & 69 & \\
\hline Palefin threadfin bream Nemipterus thosaporni & 5 & 0.42 & -0.41 & 1.73 & 3.02 & 0.90 & 0.637 & 1.032 & 70 & 70 & 70 & 70 & 70 \\
\hline Greater lizardfish Saurida tumbil & 7 & 0.29 & -0.28 & 0.46 & 3.166 & 3.50 & 0.557 & 0.748 & 71 & 71 & 71 & 71 & 7 \\
\hline Spotfin hogfish Bodianus pulchellus & 12 & 0.19 & -0.776 & 0.25 & 2.97 & 5.44 & 0.261 & 0.414 & 65 & 65 & 65 & 65 & 6 \\
\hline Bluestriped grunt Haemulon sciurus & 6 & 0.484 & -0.011 & 0.50 & 2.99 & 2.80 & 0.555 & 0.721 & 65 & 65 & 65 & 65 & 6 \\
\hline Black margate Anisotremus surinamensis & 8 & 0.174 & -0.45 & 0.374 & 3.042 & 4.62 & 0.532 & 0.749 & 65 & 65 & 65 & 65 & \\
\hline Sailors choice Haemulon parra & 7 & 0.22 & -0.355 & 0.428 & 2.993 & 3.88 & 0.583 & 0.830 & 65 & 65 & 65 & 65 & \\
\hline Tomato grouper Cephalopholis sonnerati & 9 & 0.091 & -2.095 & 0.333 & 3.208 & 4.82 & 0.397 & 0.748 & 65 & 65 & 65 & 65 & 6 \\
\hline White grunt Haemulon plumierii & 8 & 0.186 & -0.776 & 0.375 & 3.161 & 4.29 & 0.481 & 0.734 & 65 & 65 & 65 & 65 & \\
\hline Great barracuda Sphytraena barracuda & 15 & 0.172 & -0.461 & 0.20 & 3.083 & 7.07 & 0.224 & 0.310 & 65 & 65 & 65 & 65 & \\
\hline Yellowtail snapper Ocyurus chrysurus & 8 & 0.279 & -0.36 & 0.45 & 2.805 & 3.25 & 0.401 & 0.627 & 72 & 72 & 72 & 72 & 7 \\
\hline European seabass Dicentrarchus labrax ${ }^{\mathrm{b}}$ & 15 & 0.14 & -0.20 & 0.10 & 3.045 & 11.66 & 0.268 & 0.344 & 73 & 73 & 73 & 73 & 7 \\
\hline Japanese threadfin bream Nemipterus japonicus & 8 & 0.314 & -1.11 & 0.52 & 2.991 & 2.18 & 0.286 & 0.590 & 75 & 75 & 75 & 74 & 7 \\
\hline Eurasian perch Perca fluviatilis & 22 & 0.163 & -0.14 & 0.12 & 3.19 & 10.13 & 0.131 & 0.168 & 76 & 76 & 76 & 76 & \\
\hline Kamchatka flounder Atheresthes evermanni & 15 & 0.302 & -0.29 & 0.18 & 3.057 & 5.71 & 0.132 & 0.197 & 77 & 77 & 77 & 77 & 77 \\
\hline Pacific ocean perch Sebastes alutus & 98 & 0.167 & -0.907 & 0.05 & 3.03 & 13.52 & 0.000 & 0.001 & 79 & 78 & 78 & 78 & 7 \\
\hline Yellowfin sole Limanda aspera & 34 & 0.165 & 2.035 & 0.12 & 3.083 & 12.07 & 0.068 & 0.067 & 79 & 80 & 80 & 80 & 8 \\
\hline Alaska plaice Pleuronectes quadrituberculatus & 31 & 0.18 & 1.96 & 0.22 & 2.897 & 8.71 & 0.073 & 0.074 & 79 & 81 & 81 & 81 & $\delta$ \\
\hline Atka mackerel Pleurogrammus monopterygius & 15 & 0.439 & -0.13 & 0.3 & 3.091 & 3.76 & 0.075 & 0.117 & 79 & 82 & 82 & 82 & 8 \\
\hline English sole Parophrys vetulus & 22 & 0.31 & -0.751 & 0.26 & 3.127 & 4.26 & 0.040 & 0.075 & 79 & 83 & 83 & 84 & 8 \\
\hline Pacific cod Gadus macrocephalus & 7 & 0.33 & -0.15 & 0.65 & 2.89 & 2.59 & 0.501 & 0.704 & 85 & 85 & 85 & 86 & 87 \\
\hline Spiny dogfish Squalus acanthias & 66 & 0.06 & -4.79 & 0.09 & 3.004 & 13.54 & 0.027 & 0.051 & 79 & 88 & 88 & 89 & 5 \\
\hline Arrowtooth flounder Atheresthes stomias & 23 & 0.17 & -0.50 & 0.20 & 3.103 & 7.10 & 0.104 & 0.149 & 79 & 90 & 90 & 90 & 9 \\
\hline Sablefish Anoplopoma fimbria & 94 & 0.141 & -6.05 & 0.10 & 3.19 & 6.04 & 0.001 & 0.003 & 79 & 82 & 82 & 82 & 82 \\
\hline Australian gemfish ${ }^{\mathrm{c}}$ Rexea solandri & 16 & 0.196 & -0.585 & 0.458 & 3.389 & 2.01 & 0.171 & 0.256 & 92 & 91 & 91 & 91 & 9 \\
\hline
\end{tabular}

${ }^{a}$ Also known as Chilean sardine.

bAlso known as European bass Morone labrax.

${ }^{\mathrm{c}}$ Also known as silver gemfish.

References:

1. ICES (1978).

2. Burd (1978).

3. Burd (1974).

4. Wespetad (1991).

5. Carles (1974).

6. Carles Martín (1975).

7. Johnson et al. (1983).

8. Beverton and Holt (1959).

9. Moreau (1988).

10. Marr (1960).

11. Stanley (1978).

12. Finucane and Vaught (1986).

13. Vega-Cendejas et al. (1997).

14. Matthew (1960).

15. van der Elst (1981).

16. Pertierra (1987).

17. Belveze (1972).

18. Delgado et al. (1981).

19. Delgado and Fernandez (1985).

20. Holt (1960).

21. Bard (1973)

22. Priol (1944).

23. Dorel (1986)

24. Stergiou et al. (1997).

25. Suárez-Caabro and Duarte-Bello (1961).

26. Collette and Nauen (1983).

27. Moore (1951).

28. Yabuta et al. (1960).

29. Westman and Gilbert (1941).

30. Brown et al. (1991).

31. Anon (1995).
32. Nose et al. (1957).

33. Stequert and Marsac (1989).

34. Nakamura and Uchiyama (1966).

35. Beverton (1963).

36. Blackburn (1949).

37. Wada (1988).

38. Hughes (1974).

39. V. V. Sablin (unpublished).

40. Suyama et al. (1992).

41. Nall (1927, cited in Beverton and Holt 1959).

42. Pope et al. (1961).

43. Hohendorf (1966).

44. Kondo (1966).

45. Parrish and MacCall (1978).

46. Knaggs and Parrish (1973).

47. Castro Hernández and Santana Ortega (2000).

48. Stevens and Hausfield (1982).

49. Webb and Grant (1979).

50. Wine and Knaggs (1975).

51. Fitch (1956).

52. McPherson (1992).

53. Messersmith (1969).

54. Gunderson and Dygert (1988).

55. Gallardo-Cabello (1985).

56. Baxter (1967).

57. Prosch (1986).

58. Wysokinski (1986).

59. Harrison (2001).

60. Anon (2001)
61. Jones (1966).

62. Bouhlel (1975).

63. Ralston (1987)

64. Sherman et al. (1991).

65. Ault et al. (1998)

66. Moore and Labisky (1984).

67. Matheson and Huntsman (1984).

68. Jones and Jónsson (1971).

69. Pauly (1980).

70. Daniels (1987)

71. Pauly and Martosubroto (1980).

72. Johnson (1983).

73. Holden and William (1974).

74. Krishnamoorthi (1976).

75. Krishnamoorthi (1971)

76. Treasurer et al. (1992).

77. Saeger (1974).

78. Spencer et al. (2001).

79. Munk (2001).

80. Wilderbuer et al. (1992).

81. Zhang et al. (1998b).

82. Lowe et al. (2001).

83. Van Cleve and El-Sayed (1969).

84. Forrester and Ketchen (1963).

85. Karp (1982).

86. Fournier (1983).

87. Westerheim (1977).

88. Ketchen (1975)

89. Wood et al. (1979).

90. Wilderbuer and Sample (2001).

91. Rowling (1999).

92. Rowling (1997). 

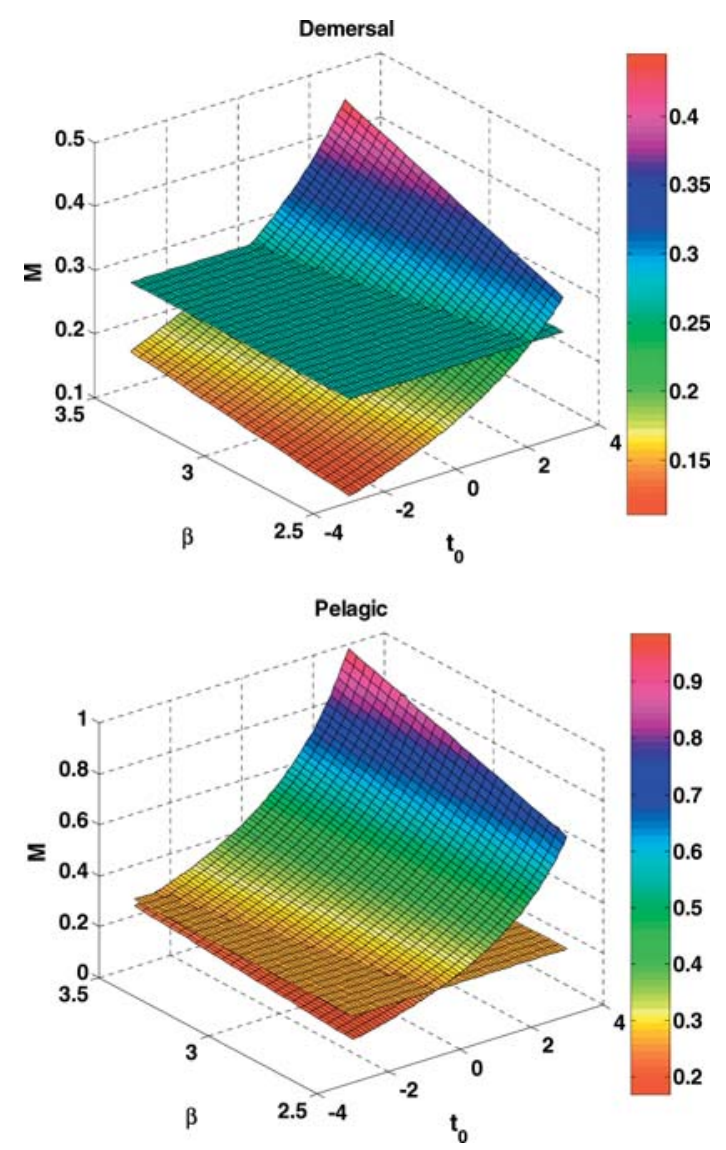

FIGURE 1.-Comparison of estimates of natural mortality $(M)$ computed from the Zhang and Megrey (ZM) model (curvilinear response surface) and the Alverson and Carney (AC) model (linear plane) for demersal and pelagic species as functions of $\beta$ and $t_{0}$. See text for additional details on these models. Values of $K=0.1$ and $t_{\max }=20$ were used in all calculations. The values of $t_{\mathrm{mb}}$ used in equations (8) (ZM model) and 10 (AC model) were calculated with the formula $t_{\mathrm{mb}}=C_{i} \cdot t_{\mathrm{max}}$. For demersal species, values of $C_{i}=0.38\left(t_{\mathrm{mb}}=\right.$ 7.6) and $C_{i}=0.44\left(t_{\mathrm{mb}}=8.8\right)$ are compared for the $\mathrm{AC}$ and $\mathrm{ZM}$ models, respectively. For pelagic species, values of $C_{i}=$ $0.38\left(t_{\mathrm{mb}}=7.6\right)$ and $C_{i}=0.302\left(t_{\mathrm{mb}}=6.04\right)$ are compared.

negative values of $t_{0}$ and there was only a slight response at positive values of $t_{0}$. At low growth rates (panel D), the patterns were the same but the percent difference was much lower. For example, when $K=1.5$ and $t_{\mathrm{mb}}=5, \hat{M}_{\mathrm{ZM}}\left(t_{0}, \beta\right)=\hat{M}_{\mathrm{ZM}}(3.0,3.5)=0.2719$, $\hat{M}_{\mathrm{AC}}(3.0,3.5)=0.00249$, and $\% \operatorname{diff}(3.0,3.5)=10,900$, but when $K=0.1$ and $t_{\mathrm{mb}}=5, \hat{M}_{\mathrm{ZM}}(3.0,3.5)=1.581$, $\hat{M}_{\mathrm{AC}}(3.0,3.5)=0.4624$, and $\% \operatorname{diff}(3.0,3.5)=241.8$. Also, the percent difference response for the lowgrowth case was much less nonlinear.
Evaluation of Relationship of $t_{\mathrm{mb}}$ to Maximum Age for Different Ecological Groups

Table 1 shows the sources used in the evaluation of the relationship of $t_{\mathrm{mb}}$ to the maximum age. The value of the ratio $t_{\mathrm{mb}} / t_{\max }$ relating the maximum age to the age at maximum biomass was 0.302 for pelagic species and 0.440 for demersal species, respectively (Table 2). The mean ratios of both subgroups were significantly different from 0.38 ( $P<0.05$ for the pelagic subgroup; $P<0.01$ for the demersal subgroup), although the pelagic subgroup had a higher sample variance than the demersal group. Moreover, the ratios between the two ecological groups showed a highly significant difference $(P<0.0001)$. Thus, the ratio for specific ecological groups should be used rather than Alverson and Carney's constant of 0.38. However, the overall grand mean (i.e., the mean obtained without splitting the data into two ecologically distinct groups) was 0.393 , which is close to the 0.38 proposed by Alverson and Carney.

Table 3 shows the results of statistical analyses comparing the $M$ values calculated from the ZM model (equation 8) and the $\mathrm{AC}$ model (equation 10). These results show that the $M$ values from the revised formula were not significantly different from those of the true values for pelagic species $(P=0.594)$ and demersal species $(P=0.570)$. However, the $M$ values from the AC model were highly significantly different from the true $M$ values in the demersal subgroup $(P<0.0001)$, although they were not significantly different from those of the true values for demersal species $(P=$ 0.335 ).

In the Australian gemfish example, we estimated the value of $M$ from equation (8) as 0.171 . The variance of $M$ was then calculated from the data in Table 4 using equation (11); the calculated variance was 0.001187 , and the $95 \%$ confidence interval was $0.171 \pm 0.068$.

\section{Discussion}

Estimates of $M$ are critically necessary for the management of commercial fisheries. Standard population dynamics techniques requiring values of $M$ are yield-per-recruit analysis, spawning-biomass-per-recruit analysis, virtual population analysis, and methods based on precautionary approaches. It is well known that these methods are extremely sensitive to errors in $M$. Two $M$-based biological reference points that are widely used to manage exploited fisheries are derived from the analyses of yield per recruit and spawning biomass per recruit (National Research Council 1998). The value of $M$, or some fraction of it, has been used in some fisheries to determine the level of fishing mortality $(F)$ that would constitute overfishing. The 

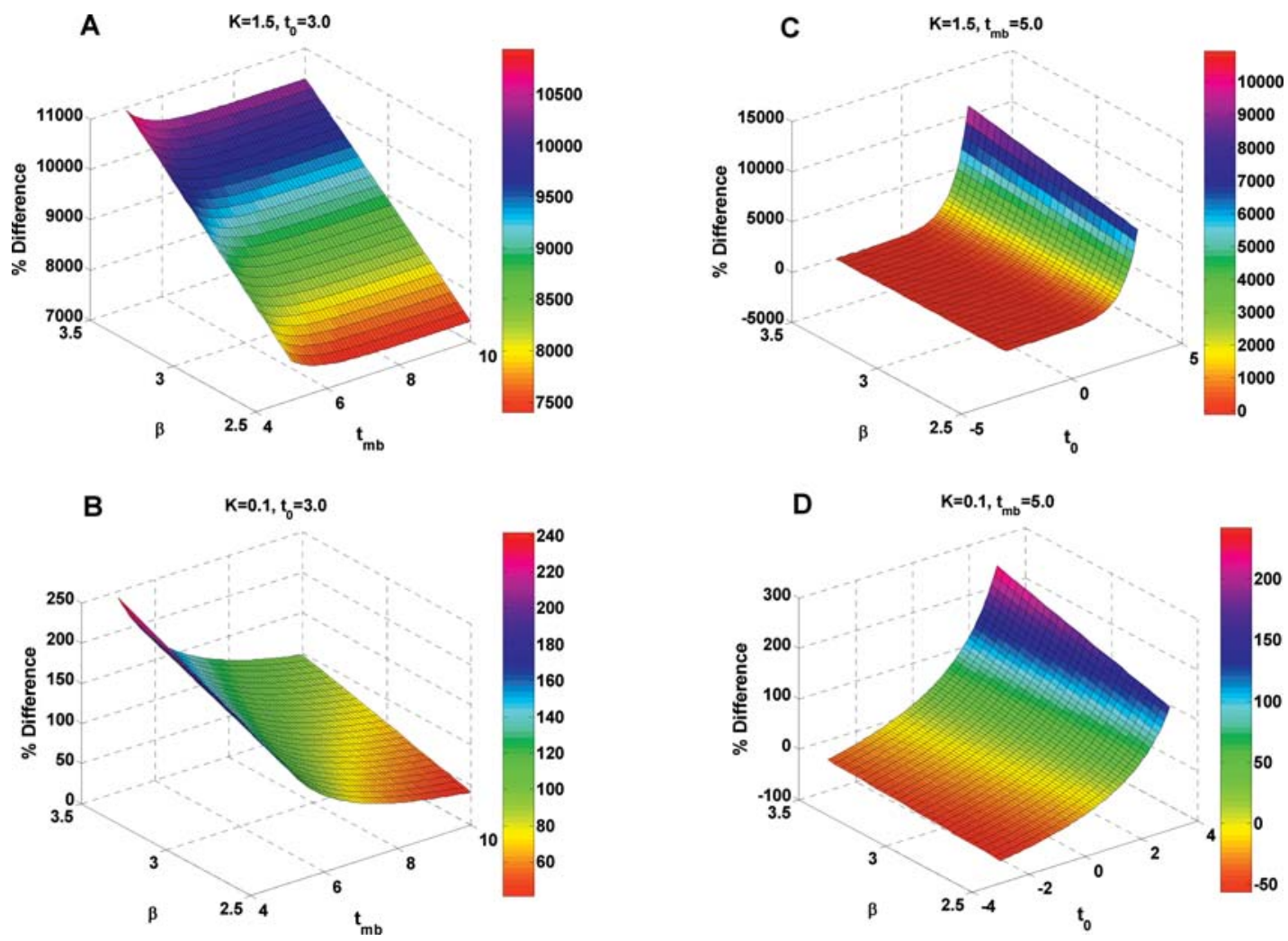

FIGURE 2.-Percent differences in estimates of $M$ from the $\mathrm{ZM}$ model and the AC model calculated from equation (12). Shown are the percent differences as a function of $\beta$ and $t_{\mathrm{mb}}$ given $t_{0}=3$ for (A) a fast-growing $(K=1.5)$ and $(\mathbf{B})$ a slow-growing fish $(K$ $=0.1$ ) and those as a function of $\beta$ and $t_{0}$ given $t_{\mathrm{mb}}=5$ for $(\mathbf{C})$ a fast-growing and (D) a slow-growing fish.

rational for this approach is that short-lived species with high values of $M$ should be able to sustain higher $F$ levels than long-lived species with low values of $M$. Indeed, many fish stocks that have sustained fisheries for long periods have sustained levels of $F$ near $M$ (Mace 1994).

Unfortunately, $M$ is difficult to estimate and it is not well known for most fish stocks. Since $M$ is rarely estimated directly in fishery assessment models, estimates, guesses, or approximate values must be used in most stock assessment models. In a comprehensive examination of catch-at-age stock assessment models, Schnute and Richards (1995) found that natural mortality is extremely difficult to estimate from standard stock assessment data. Thus, it is introduced as an assumed and fixed value. Typically, $F$ - the parameter managers are most interested in-is calculated by estimating total mortality $(Z)$ and subtracting $M$ (e.g., Ehrhardt and Ault 1992). Thus, any bias in $M$ is transferred directly into estimates of $F$. An underestimate of $M$ leads to an overestimate of $F$, and conversely for an overestimate of $M$.

Beverton and Holt (1959) and Beverton (1963) examined the utility of von Bertalanffy growth parameters in predicting $M$ and found a positive correlation between $K$ and $M$ for some major fish species. Other researchers have also proposed methods for obtaining indirect estimates of $M$ utilizing the

TABLE 2.-Mean ratio of age at maximum biomass to maximum age $\left(t_{\mathrm{mb}} / t_{\max }\right)$, variance, null hypotheses, and results of twotailed $t$-test for two ecological subgroups; $n=$ sample size; $\mu=$ mean.

\begin{tabular}{lccccrc}
\hline Subgroup & $n$ & Mean $t_{\mathrm{mb}} / t_{\max }$ & Variance & Null hypothesis & $t$-value & $P$-value \\
\hline Pelagic & 31 & 0.302 & 0.030 & $\mu_{\text {pelagic }}=0.38$ & -2.530 & $<0.05$ \\
Demersal & 60 & 0.440 & 0.021 & $\mu_{\text {demersal }}=0.38$ & 3.188 & $<0.01$ \\
Overall mean & 91 & 0.393 & 0.028 & $\mu_{\text {pelagic }}=\mu_{\text {demersal }}$ & -4.030 & $<0.0001$ \\
\hline
\end{tabular}


TABLE 3.-Results of a paired-sample $t$-test under the null hypothesis that the Zhang and Megrey (ZM) model and the Alverson and Carney (AC) model are accurate in predicting the true value of natural mortality.

\begin{tabular}{lccr}
\hline Subgroup & Statistic & ZM model & AC model \\
\hline Pelagic & $t$ & 0.539 & -0.979 \\
& $P$ & 0.594 & 0.335 \\
Demersal & $t$ & 0.571 & -4.999 \\
& $P$ & 0.570 & $<0.001$ \\
\hline
\end{tabular}

growth parameters; these include Alverson and Carney (1975), who used maximum age and $K$; Pauly (1980), who used the von Bertalanffy growth parameters $(K$, $L_{\infty}$ ) and average annual temperature; Roff (1984), who used those growth parameters and length at maturity; and Hoenig (1983), who used longevity, which is really a recasting of the Alagaraja (1984) estimator. The use of fishery-independent data will be useful to evaluate $M$ estimates for stocks in the unexploited phase or unexploited species. For example, the Ehrhardt and Ault (1992) estimator of the total mortality rate $Z$ is really estimating $M$ when the size frequency distribution is obtained by fishery-independent methods for an unexploited population.

Usually, growth parameters are the best available information in population studies for most exploited and unexploited fish stocks (Gulland 1983; Zhang 1987). Among the methods utilizing growth parameters, the AC model uses simple growth information, while others require additional information. The AC model is widely used nowadays mainly because of its minimal information requirements.

Estimates of $M$ from the AC model are most sensitive to the assumption that growth starts at $t_{0}=$ 0 when growth rates are high and to the $\beta$ and $t_{\mathrm{mb}}$ assumptions (Figures 1, 2). It was generally true that

TABLE 4.-Data and parameter estimates required for the calculation of the variance of natural mortality for Australian gemfish.

\begin{tabular}{lcl}
\hline \multicolumn{1}{r}{ Parameter } & Value & Variance \\
\hline$K^{\mathrm{a}}$ & 0.196 & 0.000016 \\
$t^{\mathrm{a}}$ & -0.585 & 0.002025 \\
$\beta^{\mathrm{b}}$ & 3.3895 & 0.0000084 \\
$t_{\text {max }}^{\mathrm{c}}$ & 16 & $2.56^{\mathrm{d}}$ \\
${\text { Demersal } t_{\text {mb }} / t_{\text {max }}}_{\text {Calculated } t_{\mathrm{mb}}}^{\text {ratio }^{\mathrm{e}}}$ & 0.440 & 0.02 \\
\hline
\end{tabular}

${ }^{a}$ Rowling (1999), Table 4.2.1 (mean of males + juveniles and females + juveniles).

${ }^{\mathrm{b}}$ Rowling (1999), Table 4.7.

${ }^{\mathrm{c} R}$ Rowling (1997).

${ }^{\mathrm{d}}$ Calculated by assuming a coefficient of variation of $10 \%$ for $t_{\max }$. ${ }^{\mathrm{e}}$ From Table 2.

${ }^{\mathrm{f}}$ Calculated from the equation $\operatorname{var}\left(t_{\mathrm{mb}}\right)=C_{i}^{2} \operatorname{var}\left(t_{\max }\right)$, where $C_{i}=$ 0.44 .
TABLE 5.-Summary statistics for model parameters. Data are from Table 1.

\begin{tabular}{lccrc}
\hline Statistic & $t_{\max }$ & $K$ & $t_{0}$ & $\beta$ \\
\hline Minimum & 2.0 & 0.05 & -6.05 & 2.57 \\
Maximum & 98.0 & 1.59 & 2.04 & 3.68 \\
Median & 13.00 & 0.18 & -0.86 & 3.01 \\
Mean & 16.21 & 0.237 & -0.99 & 3.03 \\
Variance & 232.96 & 0.037 & 1.32 & 0.032 \\
\hline
\end{tabular}

the percent difference between the two methods increased as the value of $\beta$ increased from 2.5 to 3.5 for fast- or slow-growing fishes when $t_{0}=3.0$. Figures 1 and 2 can be used to gauge the expected bias in the AC model against a particular suite of parameter values.

Frequency histograms for the model parameters were examined to see how the parameters were distributed, and summary statistics were calculated (Tables 5, 6). It can be seen that for all the species cataloged in this study $t_{0}$ is typically not zero but has a mean smaller than zero $(-0.99)$ and values that range from -6.05 to +2.04 . Similarly, $\beta$ has a mean of 3.03 and ranges from 2.57 to 3.68. Relative to the results from using Alverson and Carney's assumptions that $t_{0}=0$ and $\beta$ $=3.0$, we can see that for the ensemble average of the data the estimate of $M$ from the AC model is $15.5 \%$ higher for a long-lived, slow-growing fish and $62.5 \%$ higher for a short-lived, fast-growing fish. Also, our statistical analyses showed that the $M$ values calculated from the ZM model (equation 8) were not significantly different from the true values, while the $M$ values for the demersal subgroup calculated from the AC model were significantly different from the true values taken from the literature (Table 3). Therefore, the ZM model could be used instead of equation (10) for the estimation of $M$ since von Bertalanffy and allometric growth data are readily available for most exploited fish stocks. Also, the values of $t_{\mathrm{mb}} / t_{\max }$ for specific ecological groups could be used rather than the constant 0.38 .

In our study, the constant relating the age at maximum biomass to the maximum age varied from 0.302 to 0.440 , depending on the ecological subgroup. The variance of the constant for the pelagic subgroup

TABLE 6.-Variance-covariance matrix for model parameters.

\begin{tabular}{lcccc}
\hline Parameter & $t_{\max }$ & $K$ & $t_{0}$ & $\beta$ \\
\hline$t_{\max }$ & 232.37 & & & \\
$K$ & -0.70 & 0.04 & & \\
$t_{0}$ & -6.71 & 0.06 & 1.31 & \\
$\beta$ & -0.03 & 0.015 & 0.008 & 0.031 \\
\hline
\end{tabular}


was higher than that for the demersal group, revealing their higher variability (Table 2). Pauly (1980) also suggested the need to consider the life history features of separate ecological subgroups when estimating $M$. In particular, he suggested that the estimate of $M$ for a schooling pelagic stock be calculated by multiplying the original value of $M$ by 0.8 .

Greater attention should be paid to including estimates of $M$ in assessment models (National Research Council 1998). exploited stock dynamics can be more comprehensively analyzed by using a range of $M$ values instead of a constant value. Many studies show that fishery models are sensitive to different choices for the value of $M$. Moreover, sensitivity is influenced not only by the values chosen for $M$ but also by the interactions between $M$ and the other parameters in the models (Gulland 1983; Vetter 1988). A plausible range for $M$ could be estimated from equation (8) using the mean ratio of $t_{\mathrm{mb}} / t_{\text {max }} \pm 1 \mathrm{SD}$ (from Table 2) multiplied by $t_{\max }$ for each subgroup. A range for $M$ could also be obtained by calculating the variance using equation (11), though evaluation of that equation requires knowing the variances of four parameters and the covariances of six combinations of four parameters. The variance and covariance of the two von Bertalanffy growth parameters and the allometric length-weight parameter $\left(K, t_{0}\right.$, and $\left.\beta\right)$ can be easily obtained from routine nonlinear regression fitting algorithms. However, care should be taken when calculating the variance of $M$ using equation (11) since the delta method for approximating the variance of the function requires that the function be approximately linear (Rice 1995).

The precision of our estimate of $M$ is acceptable mainly because it is based on growth parameters estimated from length-frequency sampling data. As these data sets typically reflect large sample sizes, the parameters estimated from them tend to have low variances and high precision. This underlying precision is transferred to the precision in the estimate of $M$ through the variance equation. The value of $M$ from this analysis differs from the value included in Table 1 $\left(M_{\text {true }}=0.458\right)$, which came from a Bayesian stock assessment model (Rowling 1999). Variance estimates for $M$ could be useful in a Bayesian analysis as a starting point for the description of prior distributions for $M$.

We realize that our estimate of the variance of $M$ is an underestimate for two reasons. First, we assumed that all of the covariance terms in equation (11) were insignificant or zero. We know that this is not exactly the case and that departures from this assumption will add to the variance of $M$ depending on the correlations between the model parameters. Second, our estimate does not include process error in estimating $M$. This could be particularly relevant, as it relates to the data taken from FishBase used in the analysis. Even with these caveats, we believe that we have shown the utility of our method of calculating variances for $M$, a feature not typically available from purely empirical approaches.

In summary, we have presented an extension of the original Alverson and Carney empirical estimate of natural mortality. Our new formulation (the ZM model) is less biased than the AC model and includes easily obtainable maximum age and von Bertalanffy and allometric growth parameters. We show that the relationship between the time a stock requires to maximize its biomass and its maximum age is dependent on the ecological group to which it belongs. We also present an easy way to calculate the variance of $M$ using parameter variances usually obtainable from nonlinear regression computer algorithms.

\section{Acknowledgments}

The authors would like to thank Don Gunderson and Lee Alverson for reviewing an early draft of the paper. We also thank Jerry Ault and an anonymous reviewer for their useful comments. This research is contribution FOCI-0531 to NOAA's Fisheries-Oceanography Coordinated Investigations.

\section{References}

Alagaraja, K. 1984. Simple methods for estimation of parameters for assessing exploited fish stocks. Indian Journal of Fisheries 31:177-209.

Alverson, D. L., and M. J. Carney. 1975. A graphic review of the growth and decay of population cohorts. Journal du Conseil International pour l'Exploration de la Mer 36(2):133-143.

Anonymous. 2001. Increasing competition between fisheries and whales: Japan's whale research in the western North Pacific (JARPN II). Government of Japan, Fisheries Agency, Tokyo.

Anonymous. 1995. (No title given). International Commission for the Conservation of Atlantic Tunas. Report for biennial period, 1994-95, part I, volume 2, Madrid.

Ault, J. S., J. A. Bohnsack, and G. A. Meester. 1998. A retrospective (1979-1996) multispecies assessment of coral reef fish stocks in the Florida Keys. Fishery Bulletin 96(3):395-414.

Ault, J. S., S. G. Smith, and J. A. Bohnsack. 2005. Evaluation of average length as an indicator of exploited status for the Florida coral reef fish community. ICES Journal of Marine Science 62:417-423.

Baranov, F. I. 1918. On the question of the biological basis of fisheries. Nauchnye Issledovaniya Ikthiologicheskii Instituta Izvestiya 1:81-128. (In Russian.)

Bard, F. X. 1973. Étude sur le germon Thunnus alalunga de l'Atlantique: éléments de dynamique de populations. (A study of the albacore Thunnus alalunga in the Atlantic: 
elements of population dynamics.) International Commission for the Conservation of Atlantic Tunas Scientific Paper 3:198-224.

Baxter, J. L. 1967. Summary of biological information on the northern anchovy Engraulis mordax Girard. CAlCOFI (California Cooperative Oceanic Fisheries Investigations) Reports 1:110-116.

Belveze, H. 1972. Observations complémentaires sur le stock sardinier de l'Atlantique maroccain de 1968 à 1971 et estimation de quelques paramètres. (Supplementary observations on the sardine stock along the Moroccan Atlantic coast from 1968 to 1971 and estimation of some parameters.) Bulletin Institute of Pêches Maritime Maroco 20:5-69.

Beverton, R. J. H. 1963. Maturation, growth, and mortality of clupeid and engraulid stocks in relation to fishing. Rapports et Procès-Verbaux des Réunions, Conseil International pour l'Exploration de la Mer 154:44-67.

Beverton, R. J. H. 1992. Patterns of reproductive strategy parameters in some marine teleost fishes. Journal of Fish Biology 41:137-160.

Beverton, R. J. H., and S. J. Holt. 1957. On the dynamics of exploited fish populations. Fisheries Investigations Series II, Great Britain Ministry of Agriculture, Fisheries and Food 19.

Beverton, R. J. H., and S. J. Holt. 1959. A review of the lifespans and mortality rates of fish in nature and their relation to growth and other physiological characteristics. Pages 142-189 in G. E. W. Wolstenholme and M. O'Connor, editors. CIBA Foundation Colloquia on Aging: the lifespan of animals, volume 5. J. \& A. Churchill Ltd., London.

Blackburn, M. 1949. The age, rate of growth, and general life history of the Australian pilchard in New South Wales waters. Bulletin of the Science Industry Resource Organization 242:1-86.

Bouhlel, M. 1975. Contribution à l'étude biologique et dynamique du merlu Merluccius merluccius mediterraneus (L. 1758) du golfe de Tunis. (Contribution to the study of the biology and dynamics of the hake Merluccius merluccius mediterraneus [L. 1758] in the Gulf of Tunis.) University de Tunis, Tunis, Tunisia.

Brown, B. E., J. A. Browder, J. Powers, and C. D Goodyear. 1991. Biomass, yield models, and management of strategies of Gulf of Mexico ecosystems. Pages 125163 in K. Sherman, L. M. Alexander, and B. D. Gold, editors. Food chains, yields, models, and management of large marine ecosystems. Westview Press, Inc., Boulder, Colorado.

Burd, A. C. 1974. The northeast Atlantic herring and the failure of an industry. Pages 167-192 in F. R. Harden Jones, editor. Sea fisheries research. Paul Elek (Scientific Books), Ltd., London.

Burd, A. C. 1978. Long-term changes in North Sea herring stocks. Rapports et Procès-Verbaux des Réunions, Conseil International pour l'Exploration de la Mer 172:137-153.

Carles, C. 1974. Edad y crecimiento del bonito (Katsuwonus pelamis) y albacora (Thunnus atlanticus) en la parte occidental de Cuba. (Age and growth of bonito [Katsuwonus pelamis] and albacore in the western part of Cuba.) Resumenes de Investigación I.N.P.-C.I.P.
(Instituto Nacional de la Pesca-Centro de Investigaciones Pesqueras) 1(1):122-126.

Carles Martín, C. A. 1975. Evaluación de la pesquería de bonito en la zona occidental de Cuba. (Evaluation of the bonito fishery in the western zone of Cuba.) Revista de Investigaciones Instituto Nacional de la Pesca 1(1):7376.

Castro Hernández, J. J., and A. T. Santana Ortega. 2000. Synopsis of biological data on the chub mackerel (Scomber japonicus Houttuyn, 1782). FAO (Food and Agriculture Organization of the United Nations) Fisheries Synopsis 157.

Clark, W. J. 1999. Effects of an erroneous natural mortality rate on a simple age-structured stock assessment. Canadian Journal of Fisheries and Aquatic Sciences 56:1721-1731.

Collette, B. B., and C. E. Nauen. 1983. FAO species catalogue. volume 2. Scombrids of the world: an annotated and illustrated catalogue of tunas, mackerels, bonitos, and related species known to date. FAO (Food and Agriculture Organization of the United Nations) Fisheries Synopsis 2(125)

Daniels, R. A. 1987. Comparative life histories and microhabitat use in three sympatric sculpins (Cottidae: Cottus) in northeastern California. Environmental Biology of Fishes 19:93-110.

Delgado, A., and M. A. R. Fernandez. 1985. Datos sobre la biología de la sardina (Sardina pilchardus Walb., 1792) capturado por los cerqueros españoles en Africa Occidental de 1976 a 1982. (Data on the biology of the European pilchard [Sardina pilchardus Walb., 1792] captured by Spanish fish traps in West Africa from 1976 to 1982.) Pages 935-955 in. Simposio. Internationcal Occiedntal Africa, volume II.15 Instituto Investigaciones Pesqueras, Barcelona.

Delgado, A., M. A. R. Fernandez, and R. Goni. 1981. Contribución al estudio de la sardina (Sardina pilchardus Walb.) en aguas de Africa Occidental, III. Estudio del crecimiento por lectura directa de otolitos y por retrocalculo mediante escalimetria de escamas. (Contribution to the study of the sardine [Sardina pilchardus Walb.] in the waters of West Africa, III. A study of growth by direct reading of otoliths and through backcalculation based on scale measurements.) Boletin Instituto Español de Oceanografía (6):139-164.

Dorel, D. 1986. Poissons de l'Atlantique nord-est relations taille-poids. (Size-weight relationships among fish of the northeastern Atlantic.) Institut Francais de Recherche pour l'Exploitation de la Mer.

Efimov, Y. N. 1984. Stock condition and assessment of maximum sustainable yield of atka mackerel in the Gulf of Alaska. International North Pacific Fisheries Commission Bulletin 42:82-84.

Ehrhardt, N. M., and J. S. Ault. 1992. Analysis of two lengthbased mortality models applied to bounded catch length frequencies. Transactions of the American Fisheries Society 121:115-122.

Finucane, J. H., and R. N. Vaught. 1986. Species profile of Atlantic thread herring Opisthonema oglinum (Lesueur 1818). NOAA Technical Memorandum NMFS-SEFC182.

Fitch, J. 1956. Jack mackerel. CALCOFI (California Co- 
operative Oceanic Fisheries Investigations) Reports 1955-1956:27-28.

Forrester, C. R., and K. S. Ketchen. 1963. A review of the Strait of Georgia trawl fishery. Fisheries Research Board of Canada Bulletin 139.

Fournier, D. A. 1983. An analysis of the Hecate Strait Pacific cod fishery using an age-structured model incorporating density-dependent effects. Canadian Journal of Fisheries and Aquatic Sciences 40:1233-1243.

Frose, R., and D. Pauly, editors. 1998. FishBase 98: Concepts, design, and data sources. International Center for Living Aquatic Resources Management, Manila, Philippines.

Gallardo-Cabello, M. 1985. Análisis del crecimiento de la anchoveta Engraulis mordax Girard, en aguas de Baja California Norte (Pisces: Engraulidae). (Analysis of the growth of the anchovy Engraulis mordax Girard in the waters of Baja California Norte [Pisces: Engraulidae].) Anales del Instituto de Centro de Ciencias del Mar y Limnología 12(1):235-252.

Gulland, J. A. 1983. Fish stock assessment: a manual of basic methods. Wiley Interscience, Chichester, UK.

Gunderson, D. R. 1997. Trade-off between reproductive effort and adult survival in oviparous and viviparous fishes. Canadian Journal of Fisheries and Aquatic Sciences 54:990-998.

Gunderson, D. R., and P. H. Dygert. 1988. Reproductive effort as a predictor of natural mortality rate. Journal du Conseil International pour 1'Exploration de la Mer 44:200-209.

Harrison, T. D. 2001. Length-weight relationships of fishes from South African estuaries. Journal of Applied Ichthyology 17(1):46-48.

Hoenig, J. M. 1983. Empirical use of longevity data to estimate natural mortality rates. Fishery Bulletin 81(4):898-903.

Hohendorf, K. 1966. Eine Diskussion der von Bertalanffy Funktionen und ihre Anwendung zur Charakterisierung des Wachstums von Fischen. (A discussion of the von Bertalanffy function and its application to characterizing the growth of fish.) Kieler Meeresforschungen 22:70-97.

Holden, M. J., and T. William. 1974. The biology and population structure of bass, Dicentrarchus labrax, in English waters. Journal of the Marine Biological Association of the United Kingdom 54:91-107.

Holt, S. J. 1965. A note on the relationship between mortality rate and the duration of life in an exploited fish population. International Commission on North Atlantic Fisheries Research Bulletin 2:73-75.

Holt, S. J. 1960. A preliminary comparative study of the growth, maturity, and mortality of sardines. Pages 553561 in Rosa H Jr. and G. Murphy, editors. Proceedings of the World Scientific Meeting on the Biology of Sardines and Related Species 2(2). Food and Agriculture Organization of the United Nations, Rome.

Hughes, S. E. 1974. Stock composition, growth, mortality, and availability of Pacific saury, Cololabis saira, of the northeastern Pacific Ocean. Fishery Bulletin 72(1):121131.

ICES (International Council for the Exploration of the Sea). 1978. Report of the Working Group on Assessment of Pelagic Stocks in the Baltic. C.M. 1978/J:4, Copenhagen.

Jensen, A. L. 1996. Beverton and Holt life history invariants result from optimal trade-off of reproduction and survival. Canadian Journal of Fisheries and Aquatic Sciences 53:820-822.

Johnson, A. G. 1983. Age and growth of yellowtail snapper from South Florida. Transactions of the American Fisheries Society 112:173-177.

Johnson, A. G., A. Fable, Jr., M. L. Williams, and L. E. Barger. 1983. Age, growth, and mortality of king mackerel, Scomberomorus cavalla, from the southeastern United States. Fishery Bulletin 81(1):97-106.

Jones, B. W. 1966. The cod and the cod fishery at Faroe. Fisheries Investigations 24(5):1-32.

Jones, B. W., and J. Jónsson. 1971. Coalfish tagging experiments at Iceland. Journal of the Marine Research Institute, Reykjavik 5(1):1-27.

Karp, W. A. 1982. Biology and management of pacific cod (Gadus macrocephalus Tilesius) in Port Townsend, Washington. Doctoral dissertation. University of Washington, Seattle.

Ketchen, K. S. 1975. Age and growth of dogfish Squalus acanthias in British Columbia waters. Journal of the Fisheries Research Board of Canada 32:43-59.

Knaggs, E. H., and R. H. Parrish. 1973. Maturation and growth of Pacific mackerel, Scomber japonicus Houttuyn. California Fish and Game 59(2):114-120.

Kondo, K. 1966. Growth of Japanese mackerel, II. Age determination with use of scale. Bulletin of the Tokai Regional Fisheries Research Laboratory 45:31-60.

Krishnamoorthi, B. 1971. Biology of the threadfin bream, Nemipterus japonicus. Indian Journal of Fisheries 18(12): $1-21$

Krishnamoorthi, B. 1976. A note on mortality rates and yield per recruit in Nemipterus japonicus (Bloch). Indian Journal of Fisheries 23(1/2):252-256.

Lowe, S. A., L. W. Fritz, and H. Zenger. 2001. Atka mackerel. Section 13, pages 1-51 in Stock assessment and fishery evaluation report for the groundfish resources of the Bering Sea-Aleutian Islands regions. North Pacific Fisheries Management Council, Anchorage, Alaska.

Mace, P. M. 1994. Relationships between common biological reference points used as thresholds and targets of fisheries management strategies. Canadian Journal of Fisheries and Aquatic Sciences 51:110-122.

Marr, J. C. 1960. The causes of major variations in the catch of the Pacific sardine (Sardinops caerulea [Girard]). Proceedings of the World Scientific Meeting on the Biology of Sardines and Related Species 3:667-791.

Matheson, R. H., and G. R. Huntsman. 1984. Growth, mortality, and yield-per-recruit models for speckled hind and snowy grouper from the United States South Atlantic Bight. Transactions of the American Fisheries Society 113:607-616.

Matthew, J. 1960. Synopsis on the biology of the Southwest African pilchard (Sardinops occelata Pappé). Proceedings of the World Scientific Meeting on the Biology of Sardines and Related Species 2(2):115-135.

McPherson, G. R. 1992. Age and growth of the narrow-barred Spanish mackerel (Scomberomorus commerson Lacepède, 1800) in northeastern Queensland waters. Australian Journal of Marine and Freshwater Research 43(5):1269-1282.

Megrey, B. A., A. B. Hollowed, and R. D. Methot. 1990. 
Integrated analysis of Gulf of Alaska walleye pollock catch-at-age and research survey data using two different stock assessment procedures. International North Pacific Fisheries Commission Bulletin 50:101-120.

Messersmith, J. D. 1969. The northern anchovy (Engraulis mordax) and its fishery, 1965-1968. California Department of Fish and Game Fish Bulletin 147.

Moore, C. M., and R. F. Labisky. 1984. Population parameters of a relatively unexploited stock of snowy groupers in the lower Florida Keys. Transactions of the American Fisheries Society 113:322-329.

Moore, H. L. 1951. Estimation of age and growth of yellowfin tuna (Neothunnus macropterus) in Hawaiian waters by size frequencies. Fishery Bulletin 52(65):131-149.

Moreau, J. 1988. Estimation of natural mortality from selection and catch length-frequency data: a modification of Munro's method and application example. Fishbyte 6(2):10-12.

Munk, K. M. 2001. Maximum ages of groundfishes in waters off Alaska and British Columbia and considerations of age determination. Alaska Fishery Research Bulletin 8(1):12-21.

Nakamura, E. L., and J. H. Uchiyama. 1966. Length-weight relations of Pacific tunas. Pages 197-201 in T. A. Manar, editor. Proceedings, Governor's Conference on Central Pacific Fishery Resources, Honolulu.

Nall, G. 1927. Fisheries, Scotland, salmon fish. [Further information not available.]

National Research Council. 1998. Improving methods of fish stock assessment. National Academy Press, Washington D.C.

Nose, Y., H. Kawatsu, and Y. Hiyama. 1957. Age and growth of Pacific tunas by scale reading. Suisan Gaku Shusei: 701-716.

Parrish, R. H., and A. D. MacCall. 1978. Climatic variation and exploitation in the Pacific mackerel fishery. California Department of Fish and Game Fish Bulletin 167.

Pauly, D. 1980. On the interrelationships between natural mortality, growth parameters, and mean environmental temperature in 175 fish stocks. Journal du Conseil International pour l'Exploration de la Mer 39(3):175192.

Pauly, D., and P. Martosubroto. 1980. The population dynamics of Nemipterus marginatus (Cuvier \& Val.) off western Kalimantan, South China Sea. Journal of Fish Biology 17:263-273.

Pertierra, J. P. 1987. Crecimiento del boqueron (Engraulis encrasicolus) de la costa catalana (Mediterraneo noroccidental). (Growth of the European anchovy [Engraulis encrasicolus] along the Catalan coast [northwestern Mediterranean].) Investigación Pesquera 51:263-275.

Pope, J. A., D. H. Mills, and W. M. Shearer. 1961. The fecundity of Atlantic salmon (Salmo salar Linn.). Reports of Freshwater Salmon Fisheries Research of Scotland 26.

Priol, E. P. 1944. Observations sur les germons et les thons rouges capturés par les pêcheurs bretons. (Observations on albacore and bluefin tuna captured by Breton fishermen.) Revue des Travaux de l'Institut des Pêches Maritimes 13(1-4):384-439.

Prosch, R. M. 1986. Early growth in length of the anchovy
Engraulis capensis Gilchrist off South Africa. South African Journal of Marine Science 4:181-191.

Quinn, T. J. II, and R. B. Deriso. 1999. Quantitative fish dynamics. Oxford University Press, New York.

Quinn, T. J. II, and N. J. Szarzi. 1993. Determination of sustained yield in Alaska's recreational fisheries. Pages 61-84 in G. Kruse, D. M. Eggers, R. J. Marasco, C. Pautzke, and T. J. Quinn II, editors. Proceedings of the International Symposium on Management Strategies for Exploited Fish Populations. University of Alaska-Fairbanks, Alaska Sea Grant College Program Report 93-02, Fairbanks.

Ralston, S. 1987. Mortality rates of snappers and groupers. Pages 375-404 in J. Polovina and S. Ralston, editors. Tropical snappers and groupers: biology and fisheries management. Westview Press, Inc., Boulder, Colorado.

Rice, J. A. 1995. Mathematical statistics and data analysis, 2nd edition. Duxbury Press, Belmont, California.

Rikhter, V. A., and V. N. Efanov. 1976. On one of the approaches to estimation of natural mortality of fish populations. International Commission of North Atlantic Fisheries, Research Document 76/VI/8, Dartmouth, Nova Scotia.

Roff, D. A. 1984. The evolution of life history parameters in teleosts. Canadian Journal of Fisheries and Aquatic Sciences 41:989-1000.

Rowling, K. R. 1997. The collapse of the eastern Australian gemfish stock: issues for management and the role of fisheries science. Pages 210-214 in D. A. Hancock, D. C. Smith, A. Grant, and J. P. Beumer. Developing and sustaining world fisheries resources: the state of science and management. CSIRO Publishing, Melbourne, Australia.

Rowling, K. R. 1999. The fisheries biology and population dynamics of gemfish, Rexea solandri. Doctoral dissertation. University of New South Wales, Sydney, Australia.

Saeger, J. 1974. Der Befischungszustand der Flunderpopulation in der Kieler Bucht. (The fishery status of the flounder population in Kiel Bay.) Master's thesis. Kiel University, Kiel, Germany.

Saville, A. 1977. Survey methods of appraising fisheries resources. FAO Fisheries Technical Paper 171.

Schnute, J., and L. J. Richards. 1995. The influence of error on population estimates from catch-age models. Canadian Journal of Fisheries and Aquatic Sciences 52:2063-2077.

Seber, G. A. F. 1982. The estimation of animal abundance. MacMillan Publishing Company, New York.

Sherman, K., L. M. Alexander, and B. D. Gold. 1991. Food chains, yields, models, and management of large marine ecosystems. Westview Press, Inc., Boulder, Colorado.

Sparre, P., E. Ursin, and S. C. Venema. 1989. Introduction to tropical fish stock assessment, part 1. Manual. FAO Fisheries Technical Paper 306.

Spencer, P. D., D. H. Ito, and J. I. Ianelli. 2001. Pacific ocean perch. Section 10, pages 1-41 in Stock assessment and fishery evaluation report for the groundfish resources of the Bering Sea-Aleutian Islands regions. North Pacific Fisheries Management Council, Anchorage, Alaska.

Stanley, C. A. 1978. Area of distribution, movements, age composition, and mortality rates of the Australian salmon population in Tasmania, Victoria, and New South Wales. 
Australian Journal of Marine and Freshwater Research 29:417-433.

Stequert, B., and F. Marsac. 1989. Tropical tuna: surface fisheries in the Indian Ocean. FAO Fisheries Technical Paper 282.

Stergiou, K. I., E. D. Christou, D. Georgopoulous, A. Zenetos, and C. Souvermezoglou. 1997. The Hellenic seas: physics, chemistry, biology, and fisheries. Pages 415538 in A. D. Ansell, R. N. Gibson, and M. Barnes, editors. Oceanography and marine biology: an annual review. UCL Press, London.

Stevens, J. D., and H. F. Hausfeld. 1982. Age determination and mortality estimates on an unexploited population of jack mackerel Trachurus declivis (Jenyns, 1841) from southeast Australia. CSIRO Marine Laboratory Report 148.

Suárez-Caabro, J. A., and P. P. Duarte-Bello. 1961. Biología pesquera del bonito (Katsuwonus pelamis) y la albacora (Thunnus atlanticus) en Cuba. (Fisheries biology of skipjack tuna [Katsuwonus pelamis] and blackfin tuna [Thunnus atlanticus] in Cuba.) Instituto Cubano de Investigación, Havana.

Suyama, S., Y. Sakurai, T. Meguro, and K. Shimazaki. 1992. Estimation of the age and growth of pacific saury Cololabis saira in the central North Pacific Ocean determined by otolith daily growth increments. Nippon Suisan Gakkaishi 58(9):1607-1614.

Tanaka, S. 1960. Studies on the dynamics and the management of fish populations. Bulletin of the Tokai Regional Fisheries Research Laboratory 28. (In Japanese.)

Treasurer, J. W., R. Owen, and E. Bowers. 1992. The population dynamics of pike, Esox lucius, and perch, Perca fluviatilis, in a simple predator-prey system. Environmental Biology of Fishes 34:65-78.

Van Cleve, R., and S. Z. El-Sayed. 1969. Age, growth, and productivity of an English sole (Parophrys vetulus) population in Puget Sound, Washington. Bulletin of the Pacific Marine Fisheries Commission 7:51-72.

van der Elst, R. 1981. A guide to the common sea fishes of southern Africa. C. Struik, Cape Town.

Vega-Cendejas, M. E., G. Mexicabo-Cíntora, and A. M. Arce. 1997. Biology of the thread herring Opisthonema oglinum (Pices: Clupeidae) from a beach seine fishery of the Campeche Bank, Mexico. Fisheries Research 30:117-126.

Vetter, E. F. 1988. Estimation of natural mortality in fish stocks: a review. Fishery Bulletin 86(1):25-43.

Von Bertalanffy, L. 1938. A quantitative theory of organic growth. Human Biology 10(2):181-213.

Wada, T. 1988. Population dynamics on Japanese sardine, Sardinops melanostictus caught by the domestic purse seine fishery in the waters off the coast of southeastern Hokkaido. Bulletin of the Hokkaido Regional Fisheries Research Laboratory 52.

Wakabayashi, K., and K. Watanabe. 1990. Assessment of walleye pollock abundance of the southwest Pacific coast of Hokkaido based on catch statistics and survey data. International North Pacific Fisheries Commission Bulletin 50:279-289.

Webb, B. F., and C. J. Grant. 1979. Age and growth of jack mackerel, Trachurus declivis (Jenyns), from southeastern
Australian waters. Australian Journal of Marine and Freshwater Research 30(1):1-9.

Wespestad, V. G. 1991. Pacific herring population dynamics, early life history, and recruitment variation relative to eastern Bering Sea oceanographic factors. Doctoral dissertation. University of Washington, Seattle.

Westerheim, S. J. 1977. . Length-weight and length-girth relationships, maturity, spawning season, and diet of Pacific cod (Gadus macrocephalus) collected in British Columbia waters during April 1975-February 1976. Fisheries Research Board of Canada Manuscript Report Series 1420.

Westman, J. R., and P. W. Gilbert. 1941. Notes on the age determination and growth of the Atlantic bluefin tuna, Thunnus thynnus (Linnaeus). Copeia 1941:70-72.

Wilderbuer, T. K., and C. I. Zhang. 1999. Evaluation of the population dynamics and yield characteristics of Alaska plaice, Pleuronectes quadrituberculatus, in the eastern Bering Sea, incorporating results of an age-structured analysis. Fisheries Research 41:183-200.

Wilderbuer, T. K., G. E. Walters, and R. G. Bakkala. 1992. Yellowfin sole, Pleuronectes asper, of the eastern Bering Sea: Biological characteristics, history of exploitation, and management. Marine Fisheries Review 54(4):1-18.

Wilderbuer, T. K., and T. M. Sample. 2001. Arrowtooth flounder. Section 5, pages 1-24 in Stock assessment and fishery evaluation report for the groundfish resources of the Bering Sea-Aleutian Islands regions. North Pacific Fisheries Management Council, Anchorage, Alaska.

Wine, V., and E. Knaggs. 1975. Maturation and growth of jack mackerel, Trachurus symmetricus. California Department of Fish and Game, Marine Research Technical Report 21:1-26.

Wood, C. C., K. S. Ketchen, and R. J. Beamish. 1979. Population dynamics of spiny dogfish (Squalus acanthias) in British Columbia waters. Journal of the Fisheries Research Board of Canada 36:647-656.

Wysokinski, A. 1986. The living marine resources of the Southeast Atlantic. FAO Fisheries Technical Paper 178, revision 1.

Yabuta, Y., M. Yukinawa, and Y. Warashina. 1960. Growth and age of yellowfin tuna, 2. Age determination (scale method). Report of the Nankai Regional Fisheries Research Laboratory 12:63-74.

Zhang, C. I. 1987. Biology and population dynamics of Alaska plaice, Pleuronectes quadrituberculatus, in the eastern Bering Sea. Doctoral dissertation. University of Washington, Seattle.

Zhang, C. I. 1991. Fisheries resource ecology. Woosung Publishing Co., Seoul, Korea.

Zhang, C. I., C. S. Park, and M. H. Sohn. 1998a. A study on the stock management of the sharp-toothed eel, Muraenesox cinereus (FORKSÅL) in Korean waters, IV. Population ecological characteristics and biomass. Journal of the Korean Society of Fisheries Resources 1:2535 .

Zhang, C. I., T. K. Wilderbuer, and G. E. Walters. 1998b. Biological characteristics and fishery assessment of Alaska plaice, Pleuronectes quadrituberculatus, in the eastern Bering Sea. Marine Fisheries Review 60(4):1627. 